\title{
Charm Current-Current Correlators in Twisted Mass Lattice QCD
}

\author{
Karl Jansen \\ NIC / DESY Zeuthen \\ Platanenallee 6 \\ D-15738 Zeuthen \\ Germany \\ E-mail: Karl.Jansen@desy.de
}

\section{Marcus Petschlies*}

Humboldt-Universität zu Berlin, Institut für Physik

Newtonstraße 15

12489 Berlin

E-mail: marcuspe@physik.hu-berlin.de

\section{Carsten Urbach}

HISKP (Theory) and Bethe Center for Theoretical Physics

Rheinische Friedrich-Wilhelms-Universität Bonn

Nussalle 14-16, 53115 Bonn, Germany

E-mail: urbach@hiskp.uni-bonn.de

\section{for the European Twisted Mass collaboration}

\begin{abstract}
The current correlator method has been shown to be a practical tool to extract the charm quark mass and strong coupling constant from Lattice QCD data as an alternative to the sum rule approach using experimental electron-positron annihilation cross section data. We report on the progress of an investigation of charm current-current correlators in $N_{f}=2$ Twisted Mass Lattice QCD. Upon determining the temporal moments of the current correlators we compare to the low-energy expansion of the moments in perturbative QCD and calculate the charm quark mass and strong coupling constant and in case of the vector current correlator directly compare both methods.
\end{abstract}

The XXIX International Symposium on Lattice Field Theory, Lattice2011

July 11-16, 2011

The Village at Squaw Valley, USA

\footnotetext{
* Speaker.
} 


\section{Introduction}

The charm quark mass and strong coupling constant are fundamental parameters of the Standard Model and thus there is an interest per se in their calculation. They are essential input parameters for the calculation of processes involving charm quarks, such as inclusive radiative $B$-decays and exclusive Kaon-decays [1]. Moreover they play an important role in the estimation of CKM matrix elements and the search for new physics beyond the Standard Model [2].

Recently the HPQCD collaboration extracted the $\overline{\mathrm{MS}}$ charm quark mass and strong coupling constant using temporal moments of charmed lattice current correlators [3]. Using the highly improved staggered quark action and a Bayesian prior fitting analysis a few percent precision could be reached. Here we report on an ongoing effort to apply this method using a different fermion discretization, namely Wilson twisted mass Lattice QCD. In this work, we will not rely on any Bayesian prior in the fits of our data and it is one of our goals to understand, whether a similar accuracy can be reached as given in [3].

\section{Low momentum expansion of polarization functions in perturbative QCD}

The general strategy of the current correlator method is the non-perturbative estimation of derivatives $M_{n}$ of the polarization functions of in our case the pseudoscalar and vector currents from lattice data and to compare them to their continuum counterparts determined in perturbation theory. The derivatives are readily deduced from the momentum expansion of the polarization functions in the limit $q^{2} \ll \bar{m}_{c}^{2}$

$$
\begin{aligned}
q^{2} \Pi_{c}^{\kappa} & =i \int d^{4} x \mathrm{e}^{i q x}\left\langle 0\left|T\left\{J^{\kappa}(x) J^{\kappa}(0)\right\}\right| 0\right\rangle, \\
\left(-q^{2} g_{\mu v}+q_{\mu} q_{v}\right) \Pi_{c}^{\delta}+q_{\mu} q_{v} \Pi_{c L}^{\delta} & =i \int d^{4} x \mathrm{e}^{i q x}\left\langle 0\left|T\left\{J_{\mu}^{\delta}(x) J_{v}^{\delta}(0)\right\}\right| 0\right\rangle, \\
\Pi^{\kappa, \delta}\left(q^{2}\right) & =\frac{3}{16 \pi^{2}} \sum_{k \geq-1} \bar{C}_{k}^{\kappa, \delta} z^{k}, \\
\bar{C}_{k}^{\kappa, \delta} & =\sum_{m \geq 0}\left(\frac{\alpha_{s}}{\pi}\right)^{m} \bar{C}_{k}^{\kappa, \delta(m)}\left(\log \left(\frac{\bar{m}_{c}^{2}(\mu)}{\mu^{2}}\right)\right), \\
M_{n}^{\kappa, \delta} & =\left.\frac{12 \pi^{2}}{n !}\left(\frac{d}{d q^{2}}\right)^{n} \Pi_{c}^{\kappa, \delta}\left(q^{2}\right)\right|_{q^{2}=0}
\end{aligned}
$$

with $\delta=v, a, \kappa=p, s, J^{p}=\bar{c} \gamma_{5} c, J^{s}=\bar{c} c, J_{\mu}^{v}=\bar{c} \gamma_{\mu} c, J_{\mu}^{a}=\bar{c} \gamma_{\mu} \gamma_{5} c$. The perturbative expansion of the coefficients $\bar{C}_{k}^{\kappa, \delta}$ has nowadays reached the 4 loop level $\left(\mathscr{O}\left(\alpha_{s}^{3}\right)\right.$ ) ( cf. [4] and references therein).

\section{Lattice Formulation}

The calculation we report on here is based on gauge configurations produced by the European Twisted Mass collaboration (ETMC) using $N_{f}=2$ flavors of maximally twisted and mass degenerate Wilson fermions. We refer the reader to ref. [5] and references therein. We treat the 
charm degrees of freedom in a partially quenched framework by adding a doublet of heavy quarks $\chi=\left(\chi_{+}, \chi_{-}\right)$in the valence sector with valence quark action

$$
\mathscr{S}_{\text {val }}=\sum_{x} \bar{\chi}(x)\left(D_{W}+m_{\mathrm{cr}}+i \mu_{h} \gamma_{5} \tau^{3}\right) \chi(x)
$$

In this framework automatic $\mathscr{O}(a)$ improvement [6] is in place with the same critical Wilson mass $m_{\mathrm{cr}}$ as used in the light quark sector. For the lattice operators representing the physical charm currents for a given spin structure $\Gamma$ we have three natural choices (given in the physical basis).

$$
J_{\Gamma}^{0}=\bar{\psi} \Gamma \otimes \mathbb{1} \psi ; \quad J_{\Gamma}^{3}=\bar{\psi} \Gamma \otimes \tau^{3} \psi ; \quad J_{\Gamma}^{ \pm}=\bar{\psi} \Gamma \otimes \tau^{ \pm} \psi .
$$

At non-zero lattice spacing the correlation of these operators with themselves will give a different results for each operator due to lattice artifacts. Concerning the physical charm fields we would need to use the singlet currents and their corresponding translation in terms of the $\chi$ fields in the twisted basis. However, in our calculation we will not consider contributions from quarkdisconnected diagrams. This is not a source of error given the fact that the perturbative expressions we will compare to will not include singlet contributions as well (entering at $\mathscr{O}\left(\alpha_{s}^{3}\right)$ for the vector and $\mathscr{O}\left(\alpha_{s}^{2}\right)$ for the pseudoscalar currents). But given the absence of quark-disconnected diagrams the two-point correlator of $J_{\Gamma}^{0}$ will coincide with that of $J_{\Gamma}^{3}$. In the continuum limit vector flavor symmetry restoration will entail the latter to become equal to the correlation function of $J_{\Gamma}^{ \pm}$. This circumstance allows us to exploit the features of tmLQCD when it comes to the multiplicative renormalization of the bare current correlators to our advantage.

In terms of the currents defined above the renormalized and dimensionless vector and pseudoscalar moments read in the twisted basis

$$
\begin{aligned}
G_{n}^{V} & =Z_{V}^{2} a^{6} \sum_{t / a=-N_{t} / 2+1}^{N_{t} / 2-1}(t / a)^{n}\left\langle J_{V}^{0 / 3} J_{V}^{0 / 3}(t, \vec{p}=0)\right\rangle^{c o n n} \\
& =Z_{A}^{2} a^{6} \sum_{t / a=-N_{t} / 2+1}^{N_{t} / 2-1}(t / a)^{n}\left\langle J_{A}^{ \pm} J_{A}^{\mp}(t, \vec{p}=0)\right\rangle \\
G_{n}^{P} & =\left(2 a \mu_{h}\right)^{2}\left(\frac{Z_{S}}{Z_{P}}\right)^{2} a^{6} \sum_{t / a=-N_{t} / 2+1}^{N_{t} / 2-1}(t / a)^{n}\left\langle J_{S}^{0 / 3} J_{S}^{0 / 3}(t, \vec{p}=0)\right\rangle^{c o n n} \\
& =\left(2 a \mu_{h}\right)^{2} a^{6} \sum_{t / a=-N_{t} / 2+1}^{N_{t} / 2-1}(t / a)^{n}\left\langle J_{P}^{ \pm} J_{P}^{\mp}(t, \vec{p}=0)\right\rangle .
\end{aligned}
$$

Using $\mu_{h}^{R}=\mu_{h} / Z_{P}$ we introduced additional factors of $a \mu_{h}$ such that only the scale independent ratio $Z_{S} / Z_{P}$ is needed for the scalar moments and no renormalization factor for the pseudoscalar moments. For the scale independent renormalization factors $Z_{P} / Z_{S}, Z_{A}, Z_{V}$ we use the non-perturbative renormalization data provided by ETMC ([7] and private communication).

The ensembles we choose comprise four different lattice spacings ranging from $a \approx 0.05 \mathrm{fm}$ to $a \approx 0.1 \mathrm{fm}$ and light pseudoscalar masses in the range $280 \mathrm{MeV} \lesssim m_{P S} \lesssim 650 \mathrm{MeV}$ as well as up to two lattice volumes. For each triple $\left(a, L, m_{P S}\right)$ the current two-point functions were measured with four to seven charm valence quark masses such that the charmed meson masses $a m_{J / \psi} / a f_{P S}$, $a m_{\eta_{c}} / a f_{P S}$ and $a m_{D^{ \pm}} / a f_{P S}$ in units of the light pseudoscalar decay constant covered the physical 
value [8]. We are thus able to study the dependence of the moments $G=G\left(a, L, m_{P S}, \mu_{c}\right)$ on all lattice parameters. The values of the light pseudoscalar decay constants at the physical point for all four lattice spacings were calculated in a separate dedicated fit along the lines of [5].

\section{Analysis and results}

\subsection{General outline}

In our analysis we will model the dependence of the moments on the lattice parameters $\mu_{q}, \mu_{c}$ and $a$. For extrapolating to the physical light quark mass we shall use the charged pion mass $m_{P S}$, for interpolating to the physical charm quark mass the ground state mass determined from the $c \bar{c}$ non-singlet vector current correlator $m_{J / \psi}$ and the lattice spacing dependence will be studied using $a / f_{P S}$. Finite volume effects turn out to be negligible in the charm sector.

We shall use two methods:

- interpolate the lattice data at each value of the lattice spacing to common reference points $\left(\left(m_{P S} / f_{P S}\right),\left(m_{J / \psi} / f_{P S}\right)\right)^{\mathrm{ref}}$. This strategy we shall denote with "ref".

- perform a combined fit to our data describing the combined $\left(m_{P S} / f_{P S}, m_{J / \psi} / f_{P S}, a\right)$ dependence. This method we shall denote with "all" and it is based on splitting the fit function into a continuum part and one that models lattice artifacts as follows

$$
\begin{aligned}
\mathscr{F}\left(a f_{P S}, a m_{P S}, a m_{J / \psi}\right)= & \mathscr{F}_{\text {cont }} \times \mathscr{F}_{\text {latt }}=\sum_{i=0}^{M} \sum_{j=0}^{N}\left(a m_{P S} / a f_{P S}\right)^{2 i}\left(a m_{J / \psi} / a f_{P S}\right)^{j} \\
& \times\left(1+\sum_{\substack{0 \leq l, m, n \leq 2 \\
0<l+m+n \leq 2,4}}\left(a f_{P S}\right)^{2 l}\left(a m_{P S}\right)^{2 m}\left(a m_{J / \psi}\right)^{2 n}\right) .
\end{aligned}
$$

We then read of the value of the moments at zero lattice spacing and at the physical point by setting

$$
\left.G\right|_{\text {physical }}=\mathscr{F}_{\text {cont }}\left(m_{\pi} / f_{\pi}, m_{J / \psi} / f_{\pi}\right) .
$$

Either way we will end up with estimates for the continuum values of the moments or functions thereof. With these estimates we can then set up determining equations for the $\overline{\mathrm{MS}}$ quark mass $\bar{m}_{c}$ and the strong coupling $\alpha_{s}$ using the perturbative representation of the moments from the low momentum expansion of the polarization functions. We thus set

$$
\left.G\right|_{\text {physical }}=\sum_{l=0}^{L}\left(\frac{\alpha_{s}}{\pi}\right)^{l} \bar{C}^{(l)}\left(\bar{m}_{c}, \bar{\mu}\right)
$$

where similar to $G$ the coefficients $C^{(l)}$ will be functions of the original expansion coefficients in equation 2.2. In the two cases we consider we either use the charm quark mass or the strong coupling as input and solve the equation for the remaining quantity. Errors are estimated using a bootstrap method. 

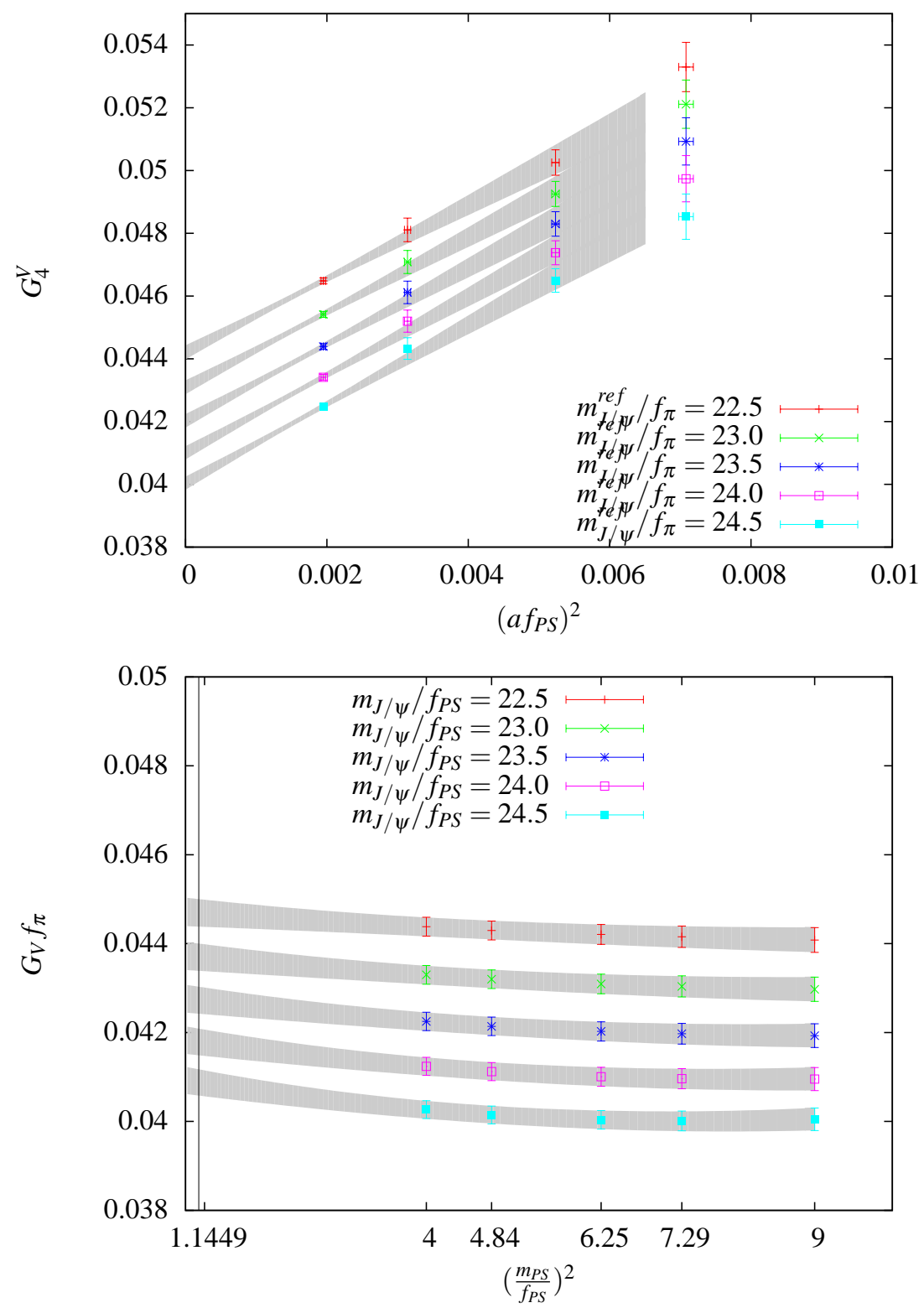

Figure 1: Example for continuum and $m_{P S}$ extrapolation of $G_{4}^{V}$ with the ref method.

\subsection{Moments from the vector current correlator}

The moments of the vector current correlator provide a benchmark of the method because their values are accessible using a dispersion integral and measurements of the hadronic cross section ratio $R(s)$. We can thus make a comparison of lattice and continuum data already on the level of individual moments. The values of the continuum moments we compare to have been provided by the authors of [9] and recently in [1] (cf. the detailed description of the extraction of the charm piece in [9]).

Taking into account all the explicit factors of the lattice spacing in equation 3.3 dimensional analysis implies the relation of dimensionless lattice moments $G_{n}^{V}$ and the corresponding continuum 


\begin{tabular}{cccc} 
No. & $f_{\pi}\left[M_{n}^{v}(2 n+2) ! /\left(12 \pi^{2}\right) / Q_{c}^{2}\right]^{1 /(2 n)}$ & $G_{2 n+2}^{V 1 /(2 n)} f_{\pi}(\mathrm{ref})$ & $G_{2 n+2}^{V 1 /(2 n)} f_{\pi}($ all $)$ \\
\hline 1 & $0.04107(32)$ & $0.04170(25)$ & $0.04146(77)$ \\
2 & $0.08792(48)$ & $0.08810(52)$ & $0.08696(87)$ \\
3 & $0.13081(60)$ & $0.13059(68)$ & $0.12945(94)$ \\
4 & $0.17106(70)$ & $0.17098(82)$ & $0.16959(102)$ \\
\hline \hline
\end{tabular}

Table 1: Comparison of continuum vector moments with results from ref and all methods.

quantities $g_{n}^{V}$ at non-zero lattice spacing

$$
G_{n}^{V}=\frac{g_{n}^{V}}{\left(a \bar{m}_{c}\right)^{n-2}}+\text { lattice artifacts . }
$$

In figure 1 we show exemplary data for the ref method: the left panel shows the continuum extrapolation of the vector moment $G_{4}^{V}$ at light pseudoscalar reference mass $a m_{P S} / a f_{P S}=2.5$ for five charm meson reference masses $a m_{J / \psi} / a f_{P S}=22.5,23.0, \ldots, 24.5$ (physical point at $m_{J / \psi} / f_{\pi}=$ 23.69(7) [8]). The reference points with lower light pseudoscalar masses than shown in the plot $\left(a m_{P S} / a f_{P S}=2.0,2.2\right)$ are not entirely covered by the data from the coarsest lattice which is why we leave it out of the extrapolation and use a linear ansatz in $a^{2}$. The right-hand side panel shows the extrapolation to the physical value of the light pseudoscalar mass $\left(a m_{P S} / a f_{P S}\right)^{\text {ref }} \rightarrow m_{\pi} / f_{\pi}=$ 1.068(3) [8]. For the second extrapolation we again use a polynomial ansatz of maximally second degree. The "all" method gives comparable results.

In table 1 we compare values our continuum extrapolated results for the four lowest lattice moments at the physical point to the continuum moments [9] (second column) determined using experimental data. Apart from the lowest moment $M_{1}^{v} / G_{4}^{V}$ we find good agreement between both the two methods and the lattice and continuum moments.

By comparing to perturbation theory we are now able to extract the $\overline{\mathrm{MS}}$ charm quark mass. To that end we use the strong coupling as an input parameter: starting from the PDG value $\alpha_{s}(\mu=$ $\left.M_{Z}, N_{f}=5\right)=0.1184$ (7) [8] we evolve it to $\alpha_{s}\left(\mu=3 \mathrm{GeV}, N_{f}=4\right)=0.255(4)$ using the RunDec program [10]. The results for the solution for the four lowest vector moments are collected in table 2. The first contribution to the uncertainty stems from the statistical error of the moment extrapolation, the physical scale $\left(f_{\pi}\right)$ and the value of $\alpha_{s}$. The second one represents the systematic uncertainty from the choice of the renormalization scale: it is obtained by matching lattice and continuum moments at $\mu=(3 \pm 1) \mathrm{GeV}$ and evolving the result back to the reference scale $\mu=$ $3 \mathrm{GeV}$ using 4-loop evolution [9].

If for each individual method we combine the quark masses from the different moments (taking into account their strong correlation) we find for the combined values

$$
\bar{m}_{c}\left(\mu=3 \mathrm{GeV}, N_{f}=3+1\right)=\left\{\begin{array}{ll}
0.979(09) \mathrm{GeV} & \text { (ref) } \\
0.998(14) \mathrm{GeV} & \text { (all) }
\end{array} .\right.
$$

The results from both extrapolation methods turn out to be compatible with the findings of reference [3], $\bar{m}_{c}\left(\mu=3 \mathrm{GeV}, N_{f}=3+1\right)=0.986(6) \mathrm{GeV}$.

A consistency check with the lowest pseudoscalar moment using the determined charm quark mass as input leads to a value of the strong coupling in good agreement with the value used as input for the charm mass. This will be discussed in detail elsewhere. 


\begin{tabular}{ccc} 
No. & $\bar{m}_{c}(\mu=3 \mathrm{GeV})[\mathrm{GeV}](\mathrm{ref})$ & $\bar{m}_{c}(\mu=3 \mathrm{GeV})[\mathrm{GeV}](\mathrm{all})$ \\
\hline 1 & $0.971(09)(01)$ & $0.979(24)(01)$ \\
2 & $0.981(10)(02)$ & $0.998(15)(02)$ \\
3 & $0.990(10)(11)$ & $1.001(12)(11)$ \\
4 & $1.014(08)(35)$ & $1.024(09)(34)$ \\
\hline \hline
\end{tabular}

Table 2: Comparison of results for the charm quark mass using ref and all extrapolated vector moments.

\section{Summary and Outlook}

With this intermediate report we showed that within the twisted mass formalism and with presently available statistics we can determine the moments of the charm vector current correlator in agreement with experimental results and with comparable uncertainty. Following two different analysis methods we can extract the $\overline{\mathrm{MS}}$ charm quark mass from both methods and find agreement taking into account both the statistical and systematic uncertainties. Yet from the comparison of the central values of both analysis methods we infer that with the presently available quality of data a systematic error of $\mathscr{O}(20) \mathrm{MeV}$ must be taken into account for the charm quark mass value.

A consistency check with the lowest pseudoscalar moment using the determined charm quark mass as input leads to a value of the strong coupling in good agreement with the value used as input for the charm mass. Currently we also investigate other methods to extract the strong coupling from flavor singlet current diagrams as recently presented in ref. [11].

As a next step it will be very interesting to apply the methods discussed here to the $N_{f}=$ $2+1+1$ gauge configurations of ETMC [12].

We thank all members of ETMC for the most enjoyable collaboration. This work is funded in part by the DFG within SFB/TR9-03. The computing time was made available to us by FZ-Jülich on JUROPA and JUGENE.

\section{References}

[1] B. Dehnadi, A. H. Hoang, V. Mateu, and S. Zebarjad, (2011).

[2] M. Antonelli et al., Phys.Rept. 494, 197 (2010).

[3] C. McNeile, C. Davies, E. Follana, K. Hornbostel, and G. Lepage, Phys.Rev. D82, 034512 (2010).

[4] Y. Kiyo, A. Maier, P. Maierhofer, and P. Marquard, Nucl.Phys. B823, 269 (2009).

[5] ETM, R. Baron et al., JHEP 1008, 097 (2010).

[6] R. Frezzotti and G. Rossi, JHEP 0408, 007 (2004).

[7] ETM, M. Constantinou et al., JHEP 1008, 068 (2010).

[8] Particle Data Group, K. Nakamura et al., J.Phys.G G37, 075021 (2010).

[9] J. H. Kuhn, M. Steinhauser, and C. Sturm, Nucl.Phys. B778, 192 (2007).

[10] K. Chetyrkin, J. H. Kuhn, and M. Steinhauser, Comput.Phys.Commun. 133, 43 (2000).

[11] K. G. Chetyrkin, Talk at the 15th Meeting of the SFB/TR 9, DESY/Zeuthen May 2011.

[12] R. Baron et al., JHEP 1006, 111 (2010). 